\title{
Integrierte Versorgung: Mehr oder weniger Staat?
}

\author{
Urs Zanoni ${ }^{a}$, Peter Berchtold ${ }^{b}$ \\ ${ }^{a} \mathrm{MPH}$, Geschäftsführer fmc Schweizer Forum für Integrierte Versorgung \\ b PD Dr. med., Präsident fmc Schweizer Forum für Integrierte Versorgung
}

\begin{abstract}
Bund, Kantone und Gemeinden haben in der Gesundheitsversorgung vielfältige und teilweise konkurrierende Rollen: Regulator, Finanzierer, (Mit-)Besitzer von Spitälern oder Pflegeheimen. Gleichzeitig werden sie immer aktiver in der Integrierten Versorgung. Und in Zukunft? Sind neue Koalitionen gefragt, die einerseits unternehmerische Freiheiten begünstigen, anderseits die Sicherheit und Qualität der Versorgung gewährleisten.
\end{abstract}

Gesundheitsversorgung und Staat: Da sind wir in der Schweiz grundsätzlich skeptisch, wittern rasch Staatsmedizin oder befürchten zumindest überbordende Bürokratie. Fast jeder hat schon von monatelangen Wartezeiten oder unterlassenen Therapien in staatlichen Gesundheitssystemen wie England, Schweden

\section{Selbstverständlich können staatliche bzw. zentralistische Gesundheitssysteme kein Vorbild sein für die Schweiz.}

oder Dänemark gehört. Vieles ist stark übertrieben, anderes falsch: Gerade die genannten Gesundheitssysteme sind uns in zentralen Bereichen wie Nutzen- und Performance-Orientierung, Integrierten Patientenpfaden und durchgehend verfügbaren Behandlungsdaten weit voraus; das bezeugen unter anderem internationale Vergleiche der OECD.

Selbstverständlich können staatliche bzw. zentralistische Gesundheitssysteme kein Vorbild sein für die Schweiz. Zu stark ist unser System von Föderalismus und Subsidiarität geprägt. Gleichzeitig gilt es, wichtige Entwicklungen der Medizin und Versorgung nicht ausser Acht zu lassen: zum Beispiel, wie bedeutsam die Zunahme an hochaltrigen, multimorbiden, chronisch kranken Menschen ist. Diese benötigen nicht nur medizinisch-pflegerische Betreuung. Genauso wichtig sind ihre psychosozialen Bedürfnisse, ausserdem juristische Aspekte (Urteilsfähigkeit der Betroffenen) und finanzielle (Ergänzungsleistungen, Hilflosenentschädigung). Aspekte also, die im Verantwortungsbereich von Kantonen und Gemeinden liegen und diese erheblich belasten
Bund, Kantone und Gemeinden sind laufend mit Interessenkonflikten konfrontiert: hin- und hergerissen zwischen dem Wohlergehen der Bevölkerung und ihren vielfältigen, teils konkurrierenden Zuständigkeiten in der Gesundheitsversorgung als Regulator, Finanzierer und (Mit-)Besitzer von Leistungserbringern. Es erstaunt deshalb nicht, dass Bund, Kantone und Gemeinden stark an integrierten, bevölkerungsorientierten Versorgungskonzepten interessiert sind, welche diese Interessenkonflikte reduzieren können.

\section{Das Medizinisch-Pflegerische und das Soziale wachsen zusammen}

Das zeigt auch die Entwicklung: Bund, Kantone und Gemeinden nehmen seit einigen Jahren deutlich aktivere Rollen in der Integrierten Versorgung ein. Davon zeugen zahlreiche Aktivitäten, zum Beispiel das Projekt «Koordinierte Versorgung» des Bundes, das «Programme cantonal Diabète» im Kanton Waadt oder

Es ist davon auszugehen, dass sich Bund, Kantone und Gemeinden in Zukunft noch viel stärker für die bessere Vernetzung der Versorgung einsetzen.

vielfältige Initiativen für regionale und lokale Versorgungnetze in allen Landesteilen, die von Gemeinden ausgehen oder an denen Gemeinden beteiligt sind. Es ist zu erwarten, dass sich Bund, Kantone und Gemeinden in Zukunft noch viel stärker für die bessere Vernetzung der Versorgung einsetzen. Denn mit der Zunahme an hochaltrigen, multimorbiden, chronisch 
kranken Menschen wachsen das Medizinisch-Pflegerische und das Soziale zusammen - was besonders Kantone und Gemeinden betrifft. Doch wie wird das vermehrte Engagement aussehen? Wie werden diese neuen Rollen neben den bisherigen Funktionen ausgeübt? Und was sind die Folgen für Leistungserbringer und Krankenversicherer? Was könnte sich für die Patientinnen und Patienten ändern?

\section{Public Governance bewegt sich im Grundsatz zwischen zwei Polen: dem Staatsversagen und dem Marktversagen.}

Um diese Fragen beantworten zu können, gilt es zuerst, die möglichen Rollen der öffentlichen Hand in den Blick zu nehmen. Public Governance, so der gängige Begriff dafür, bewegt sich im Grundsatz zwischen zwei Polen: dem Staatsversagen und dem Marktversagen [1]. In diesem Spannungsfeld bestehen vier grundsätzliche staatliche Steuerungsmodelle [2]:

- das Bereitstellen von Versorgungsleistungen durch den Staat selbst,

- die direkte Steuerung durch Regulierung,

- die indirekte Steuerung durch finanzielle Anreize,

- die Beeinflussung durch Information.

\section{Innovationen dank Bürgernähe}

Fast wichtiger als diese Unterscheidung ist die Tatsache, dass Auseinandersetzungen zur Rolle von Bund, Kantonen und Gemeinden in der Gesundheitsversorgung vielfach mit politischen Haltungen oder gar ideologisch aufgeladen sind: Wer Solidarität und offenen Zugang zu Gesundheitsleistungen als hohen Wert betrachtet, wird sich für ein staatliches Bereitstellen von Versorgungsleistungen einsetzen. Wer Wettbewerb und Konkurrenz als wichtigste Steuerungsgrundlage sieht, wird gerade umgekehrt argumentieren.

\section{Wer Solidarität und offenen Zugang zu}

Gesundheitsleistungen als hohen Wert sieht, wird sich für ein staatliches Bereitstellen von Versorgungsleistungen einsetzen.

Die Steuerungsbemühungen der öffentlichen Hand haben sich in letzter Zeit stark verlagert: Galt früher das Interesse vor allem akuten Krankheiten und der hochspezialisierten Versorgung, ist der Fokus zunehmend auf chronische Krankheiten und polymorbide Patienten gerichtet. Das ist besonders bedeutsam, weil dies auch zentrale Ziele der Integrierten Versorgung sind. Beispiel Langzeitpflege, für die in zahlreichen Kantonen die Gemeinden verantwortlich sind: Viele $\mathrm{Ge}$ -

\section{Nationales Symposium Integrierte}

\section{Versorgung am 13. Juni 2018 in Bern}

Bund, Kantone und Gemeinden werden immer wichtiger für die bessere Vernetzung und Koordination in der Gesundheitsversorgung. Was heisst das für Leistungserbringer, Krankenversicherer und Patienten? Das Symposium 2018 des fmc Schweizer Forum für Integrierte Versorgung liefert Antworten, Diskussionen und Perspektiven.

Im September 2017 hat das fmc die Hochschule Luzern - Wirtschaft mit dem Forschungsmandat «Aktuelle und künftige Rollen von Bund, Kantonen und Gemeinden in der integrierten Versorgung" beauftragt. Die Ergebnisse werden am Nationalen Symposium 2018 am 13. Juni im Berner Kursaal präsentiert und diskutiert. Ausserdem die Szenarien, die aus diesen Ergebnissen abgeleitet wurden. Alle weiteren Informationen sowie die Mög lichkeit, sich anzumelden, sind auf www.fmc.ch zu finden.

Das Symposium ist vom SIWF als Fortbildungsveranstaltung anerkannt (5 Credits).

meinden spannen zusammen und stimmen die ambulanten und die stationären Angebote so aufeinander $a b$, dass die betroffenen Menschen möglichst lange in ihrer vertrauten Umgebung leben können. Das entspricht den Patientenbedürfnissen und dämpft in der Regel das Kostenwachstum. Und häufig werden für solche Überlegungen weitere Leistungserbringer wie niedergelassene Ärzte, Apotheker, das Spital (falls vorhanden), Fachorganisationen oder die eigenen Sozialdienste eingebunden.

\section{Kantone als Entwicklungslabors}

Ein anderes Beispiel ist die medizinische Grundversorgung, die heute ein wichtiger Standortfaktor ist, besonders in ländlichen Gebieten und seit vielerorts Hausarztpraxen ersatzlos schliessen. Einzelne Gemeinden greifen ganz direkt ins Geschehen ein, indem sie (finanzielle) Anreize schaffen für Praxisübergaben oder die Eröffnung einer neuen Praxis. Andere wiederum denken regional, holen andere Gemeinden sowie Leistungserbringer an einen Tisch und entwickeln neue Versorgungsmodelle, welche die Last auf mehrere Schultern verteilen (Spitalärzte, Apotheker, Pflegeexpertinnen etc.).

Das dritte Beispiel liefert ein Expertenbericht des Bundes bzw. der Vorschlag, staatliche Globalbudgets als mögliche Massnahme zur Kostendämpfung einzuführen [3]. Wegen des lautstarken Aufschreis zahlreicher Interessengruppen ging völlig unter, dass Globalbudgets nicht grundsätzlich schlecht sein müssen. Die Capitation-Verträge von Ärztenetzen haben seit jeher den Charakter eines Globalbudgets und zeigen, dass solche Budgets viel differenzierter betrachtet werden sollten als ein simpler Gesamtbetrag, nach dessen Er- 
reichen ein Leistungs- bzw. Vergütungsstopp wie eine Guillotine herunterfällt. Zumal die Ärztenetze seit vielen Jahren gut damit fahren - gleich wie die Patientinnen und Patienten. Folglich wäre es angemessen, auch mit Blick auf die verhärteten Tarifdiskussionen, zumindest darüber zu diskutieren, ob sich staatlich verordnete Globalbudgets patienten- und leistungserbringerfreundlich ausgestalten lassen.

\section{In der Westschweiz ist die Vernetzung des medizinisch-pflegerischen Sektors mit dem Sozialbereich viel weiter fortgeschritten als in der Deutschschweiz.}

Die kantonale Ebene schliesslich, hauptverantwortlich für die Sicherung der Gesundheitsversorgung, zeichnet sich dadurch aus, dass integrierte Lösungen von Kanton zu Kanton und vor allem zwischen den Landesteilen stark variieren können. So gibt es in der Westschweiz erfolgreiche staatliche Programme zur koordinierten Versorgung von bestimmten Patientengruppen, zum Beispiel das «Programme cantonal Diabète" in der Waadt oder "Mieux vivre avec une BPCO» im Wallis. Ebenso ist in der Westschweiz die Vernetzung des medizinisch-pflegerischen Sektors mit dem Sozialbereich viel weiter fortgeschritten als in der Deutschschweiz, zum Beispiel im «Projet de Réseau Intégré de Soins aux Malades» in Genf. In der Deutsch- schweiz dagegen basieren erste Ansätze wie das Koordinationszentrum für Gesundheit und Soziales «CareNet+» im Kanton Zürich auf privater Initiative.

\section{Fazit}

Ob Bund, Kantone oder Gemeinden: Die Integration von Medizin, Pflege und Sozialem macht für die wichtigste Patientengruppe der Zukunft - alte, multimorbide, chronisch kranke Menschen - in hohem Masse Sinn, genauso für alle Akteure. Und Integrierte Versorgung wird immer eine (intelligente) Mischung aus staatlicher Governance und privaten Initiativen sein. Raum für Innovationen gibt es genug, sei es bei der Behandlung und Betreuung von Patientinnen und Patienten, sei es bei deren Befähigung zu (mehr) Eigenverantwortung, sei es bei der Finanzierung und der Vergütung.

Literatur

1 Schedler K. Public Management und Public Governance. In: Benz A, Lütz S, Schimank U, Simonis G (Hrsg.). Handbuch Governance. Theoretische Grundlagen und empirische Anwendungsfelder, Wiesbaden: VS Verlag für Sozialwissenschaften, 2007, 253-68

2 Braun D, Giraud O. Steuerungsinstrumente. In: Schubert K, Bandelow, N. (Hrsg.). Lehrbuch der Politikfeldanalyse: Oldenbourg Verlag, München - Wien 2003, 147-73

3 Bericht der Expertengruppe «Kostendämpfungsmassnahmen zur Entlastung der obligatorischen Krankenpflegeversicherung» vom 24. August 2017. (https://www.bag.admin.ch/bag/de/home/ themen/versicherungen/krankenversicherung/ kostendaempfung-kv.html) 